\title{
RÉGIS MONNEAU
}

\section{On the regularity of a free boundary for a nonlinear obstacle problem arising in superconductor modelling}

Annales de la faculté des sciences de Toulouse $\sigma^{e}$ série, tome 13, $\mathrm{n}^{\mathrm{o}} 2$ (2004), p. 289-311

<http://www.numdam.org/item?id=AFST_2004_6_13_2_289_0>

(C) Université Paul Sabatier, 2004, tous droits réservés.

L'accès aux archives de la revue «Annales de la faculté des sciences de Toulouse » (http://picard.ups-tlse.fr/ annales/) implique l'accord avec les conditions générales d'utilisation (http://www.numdam.org/conditions). Toute utilisation commerciale ou impression systématique est constitutive d'une infraction pénale. Toute copie ou impression de ce fichier doit contenir la présente mention de copyright.

\section{Numdam}

Article numérisé dans le cadre du programme Numérisation de documents anciens mathématiques http://www.numdam.org/ 


\title{
On the regularity of a free boundary for a nonlinear obstacle problem arising in superconductor modelling ${ }^{(*)}$
}

\author{
RÉGIS MONNEAU ${ }^{(1)}$
}

\begin{abstract}
We study the free boundary of solutions to a class of nonlinear obstacle problems. This class of problems contains a particular model derived from the Ginzburg-Landau equation of superconductivity. We consider solutions in a Lipschitz bounded open set $\Omega$ and prove the regularity of the free boundary when it is close enough to the fixed boundary $\partial \Omega$. We also give a result of stability of the free boundary and give a bound on the Hausdorff measure of the free boundary.
\end{abstract}

RÉSUMÉ. - Nous étudions les frontières libres asociées à des solutions d'une classe de problèmes de l'obstacle non linéaires. Cette classe de problèmes contient un modèle particulier dérivé des équations de GinzburgLandau de la supraconductivité. Nous considérons des solutions dans un ouvert borné $\Omega$ à bord Lipschitz, et nous prouvons que la frontière libre est régulière lorsque celle-ci est suffisamment proche du bord fixe $\partial \Omega$. Nous prouvons aussi un résultat de stabilité de la frontière libre et donnons une borne a priori sur la mesure de Hausdorff de cette frontière libre.

\section{Introduction}

In this article we are interested in solutions to a nonlinear obstacle problem. This problem is motivated by a work of Chapman, Rubinstein, Schatzman [13] where a model is formally derived from the Ginzburg-Landau theory for a superconductor with a density of vortices in an interior region

(*) Reçu le 14 avril 2003, accepté le 19 février 2004

(1) CERMICS, Ecole Nationale des Ponts et Chaussées, CERMICS, 6 et 8 avenue Blaise Pascal, Cité Descartes, Champs-sur-Marne, 77455 Marne-la-Vallée Cedex 2, France.

E-mail: monneau@cermics.enpc.fr 
whose boundary is a free boundary. A rigorous derivation of this model has been done by Sandier, Serfaty [23]. Se also [4, 12, 25, 24] for some related works on the mathematical analysis of superconductivity. Here we will prove rigorous results on the regularity of the free boundary contained in a Lipschitz domain.

The core of the technical part of this article is an adaptation in the framework of the nonlinear obstacle problem on non-smooth domains of Caffarelli-type techniques $[8,9]$ originally developed for linear obstacle problems on smooth domains.

The model that we consider in this paper is a nonlinear obstacle problem in a Lipschitz bounded open set $\Omega \subset \mathbf{R}^{n}$. We are interested in the minimization of the energy

$$
E(u)=\int_{\Omega} F\left(|\nabla u|^{2}\right)+u^{2}
$$

on the convex set

$$
K_{\lambda}=\left\{u \in H^{1}(\Omega), \quad u \geqslant \lambda \quad \text { on } \Omega, \quad u=\lambda_{0} \quad \text { on } \partial \Omega\right\}
$$

where $0 \leqslant \lambda \leqslant \lambda_{0}$ are two constants. We make the following assumption (which implies that the energy $E$ is strictly convex)

(A0) $F$ is a $C^{\infty}$ convex function satisfying $F^{\prime}(0)=1$ and $\lim _{q \rightarrow+\infty} F^{\prime}(q)<+\infty$.

It is classical that for each $\lambda$ there exists a unique minimizer $u_{\lambda}$ of the energy $E$ on $K_{\lambda}$. For such a minimizer the coincidence set is

$$
\{u=\lambda\}
$$

and the free boundary is

$$
\partial\{u=\lambda\}
$$

When the free boundary $\partial\{u=\lambda\}$ is smooth, the solution $u$ satisfies the following Euler-Lagrange equation

$$
\left\{\begin{array}{l}
\operatorname{div}\left(F^{\prime}\left(|\nabla u|^{2}\right) \nabla u\right)=u \quad \text { on } \quad \Omega \backslash\{u=\lambda\} \\
u=\lambda_{0} \quad \text { on } \quad \partial \Omega \\
u=\lambda \mid \quad \text { on } \quad \partial\{u=\lambda\} \\
\frac{\partial u}{\partial n}=0
\end{array}\right.
$$


Although there are two boundary conditions on the free boundary, the problem is not overdeterminated. These two boundary conditions allow to characterize the free boundary $\partial\{u=\lambda\}$ which is an unknown in this problem. We refer the reader to the monographs $[17,14,22]$ for a presentation of the classical results on the free boundary of the obstacle problem.

\subsection{Main results}

Our main result (for a smooth open set and in the linear case) is the following :

THEOREM 1.1 (Regularity transfer from the fixed boundary to the free boundary). - Let us assume that the open set $\Omega$ is smooth, and that $F(q)=$ $q$, then the energy $E$ has a unique minimizer $u_{\lambda}$ on $K_{\lambda}$ for all $\lambda \in\left[0, \lambda_{0}\right]$. Moreover there exists $\delta>0$ such that for all $\lambda \in\left(\lambda_{0}-\delta, \lambda_{0}\right)$, the free boundary $\partial\left\{u_{\lambda}=\lambda\right\}$ is a $C^{\infty}(n-1)$-dimensional manifold homeomorphic to $\partial \Omega$.

Although this result seems very natural, it was an open problem (even in this linear case), that we solve here applying the approach of blow-ups developed by Caffarelli [8] for the regularity of the free boundary of the obstacle problem. Under the assumption that $\partial \Omega \in C^{\infty}$, a nonlinear variant of theorem 1.1 was proved in [5] by A. Bonnet and the author, using the Nash-Moser inverse function theorem in dimension 2. This Nash-Moser approach could work in fact in any dimensions, but it can not be applied to a fixed boundary $\partial \Omega$ less regular than $C^{\infty}$. On the contrary the approach of Caffarelli [8] allows to deal with non-smooth fixed boundaries $\partial \Omega$.

We extend theorem 1.1 to Lipschitz open set $\Omega$ and for general convex functions $F$ satisfying assumption (A0). More precisely we make the following two assumptions on the regularity of $\Omega$ :

\section{(A1) Exterior sphere condition:}

There exists $r_{0}>0$ such that for every point $X_{0}$ of the boundary $\partial \Omega$, there exists a point $X_{1} \in \mathbf{R}^{n}$, such that the ball $B_{r_{0}}\left(X_{1}\right)$ is included in $\mathbf{R}^{n} \backslash \Omega$ and is tangent to $\partial \Omega$ at $X_{0}$.

\section{(A2) Interior cone condition:}

There exist $r_{0}>0$ and an angle $\alpha_{0} \in\left(0, \frac{\pi}{2}\right)$ such that for every point $X_{0}$ of the boundary $\partial \Omega$, there exists a unit vector $\nu \in \mathbf{S}^{n-1}$, such that $\Omega$ 
contains the cone

$$
\left\{X \in B_{r_{0}}\left(X_{0}\right), \quad<\frac{X-X_{0}}{\left|X-X_{0}\right|}, \nu>\geqslant \cos \alpha_{0}\right\}
$$

where $\langle\cdot, \cdot\rangle$ is the usual scalar product. Theorem 1.1 is a corollary of the following more general result:

THEOREM 1.2 (Regularity transfer from a Lipschitz fixed boundary). - Under assumptions (AO)-(A1)-(A2), the energy $E$ has a unique minimizer $u_{\lambda}$ on $K_{\lambda}$ for all $\lambda \in\left[0, \lambda_{0}\right]$. Moreover there exists $\delta>0$ such that for all $\lambda \in\left(\lambda_{0}-\delta, \lambda_{0}\right)$, the free boundary $\partial\left\{u_{\lambda}=\lambda\right\}$ is $a C^{\infty}(n-1)$ dimensional manifold homeomorphic to $\partial \Omega$.

In the application that we have in mind, namely a nonlinear free boundary problem arising in the description of superconductors in dimension two (see Bonnet, Monneau [5], Berestycki, Bonnet, Chapman [2]), the function $F_{0}$ is analytic convex but only defined on $\left[0, \frac{4}{27}\right)$ by $F_{0}^{\prime}(0)=1$ and

$$
h=\left(1-v^{2}\right) v \Longleftrightarrow v=F_{0}^{\prime}\left(h^{2}\right) h
$$

Using a $L^{\infty}$ control on the gradient of the solution we deduce the following result in this particular case:

COROllary 1.3 (Application to a superconducting model). - Under assumption (A1)-(A2), with $F=F_{0}$, there exists $\delta>0$ such that $\forall \lambda \in\left(\lambda_{0}-\right.$ $\left.\delta, \lambda_{0}\right)$, there exists a unique solution $u_{\lambda}$ minimizer of $E$ on $K_{\lambda}$ satisfying $\sup _{\bar{\Omega}}\left|\nabla u_{\lambda}\right|^{2}<\frac{4}{27} ;$ moreover the free boundary $\partial\left\{u_{\lambda}=\lambda\right\}$ is a $C^{\infty}(n-1)$ dimensional manifold homeomorphic to $\partial \Omega$.

Let us mention that part of the methods of [20] could be adapted to this model of superconductivity to get informations on the singularities of the free boundary when $\lambda<\lambda_{0}-\delta$.

We also prove a result on the perturbation (locally in space) of the free boundary.

THEOREM 1.4 (Local stability of the free boundary). - We assume (A0)-(A1)-(A2). Let $\lambda^{*} \in\left(0, \lambda_{0}\right)$ be such that there exists a minimizer $u_{\lambda^{*}}$ of the energy $E$ on $K_{\lambda^{*}}$ with a free boundary $\partial\left\{u_{\lambda^{*}}=\lambda^{*}\right\}$ which is $C^{\infty}$ in a compact set $\mathcal{K}^{*}$ of $\Omega$. Then for every smaller compact set $\mathcal{K} \subset \subset \mathcal{K}^{*}$ there exists $\varepsilon>0$ such that for every $\lambda$ satisfying $\left|\lambda-\lambda^{*}\right|<\varepsilon$, the unique solution $u_{\lambda}$ has a free boundary $\partial\left\{u_{\lambda}=\lambda\right\}$ which is $C^{\infty}$ in $\mathcal{K}$. 
The proof of this result is based on a geometric criterion for the regularity of the free boundary given by Caffarelli in [8] and on the continuity of the map $\lambda \mapsto u_{\lambda}$. We also refer to the book of Rodrigues [22] for classical results on the global stability of the free boundary.

Finally we give a bound on the Hausdorff measure of the free boundary, generalizing to non-smooth fixed boundaries $\partial \Omega$, a result of Brezis, Kinderlehrer [6] based on fine estimates for variational inequalities. Here the proof is an adaptation of the work of Caffarelli [9], developed for linear equations.

THEOREM 1.5 (Bound on the Hausdorff measure of the free boundary). Under assumptions (A0)-(A1)-(A2), there exists a constant $C>0$ only depending on $\Omega, \lambda_{0}, F$ such that for any minimizer $u_{\lambda}$ of $E$ on $K_{\lambda}$ with $\lambda \in\left[0, \lambda_{0}\right]$, we have

$$
\mathcal{H}^{n-1}\left(\partial\left\{u_{\lambda}=\lambda\right\}\right) \leqslant C
$$

\section{Some known results on blow-up limits}

\subsection{The simple blow-up limit}

To prove regularity results on the free boundary, the main tool (first introduced for the obstacle problem by Caffarelli in [10]) is the notion of blow-up.

Let us consider a solution $u$ to

$$
\left\{\begin{array}{l}
\Delta u=f \geqslant 1 \quad \text { on } \quad\{u>0\} \cap \Omega \\
u \geqslant 0 \text { on } \Omega \text { and }\left|D^{2} u\right|_{L^{\infty}(\Omega)} \leqslant M
\end{array}\right.
$$

with $f \in C^{0, \alpha}(\Omega)$ and $f(0)=1$. We assume that $X_{0}$ is a point of the free boundary $\partial\{u=0\}$. Let us consider the following blow-up sequence of functions

$$
u^{\varepsilon}(X)=\frac{u\left(X_{0}+\varepsilon X\right)}{\varepsilon^{2}}
$$

By assumptions, $u^{\varepsilon}(0)=\nabla u^{\varepsilon}(0)=0$ and the second derivatives $\left|D^{2} u^{\varepsilon}\right|$ are bounded by a constant independent on $\varepsilon>0$. By Ascoli-Arzela theorem, up to extraction of a convergent subsequence $\left(\varepsilon^{\prime}\right)$, we get

$$
u^{\varepsilon^{\prime}} \longrightarrow u^{0} \quad \text { uniformly on compact sets of } \mathbf{R}^{n}
$$

This function $u^{0}$ is called a blow-up limit of the function $u$ at the point $X_{0}$. 
In any dimensions, the main result for blow-up limits is the following

TheOREM 2.1 (Caffarelli [10, 8, 11], Weiss [26]; Characterization of a Simple Blow-up Limit). - The blow-up limit $u^{0}$ is unique and only depends on the point $X_{0}$ on the free boundary.

Moreover either $X_{0}$ is a singular point and then $u^{0}$ is a quadratic form, i.e.

$$
u^{0}(X)=\frac{1}{2} \quad{ }^{t} X \cdot Q_{X_{0}} \cdot X \geqslant 0
$$

where $Q_{X_{0}}$ is a symmetric matrix $n \times n$ such that $\operatorname{tr} Q_{X_{0}}=1$.

Or $X_{0}$ is a regular point and then there exists a unit vector $\nu_{X_{0}} \in \mathbf{S}^{n-1}$ such that

$$
u^{0}(X)=\frac{1}{2}\left(\max \left(<X, \nu_{X_{0}}>, 0\right)\right)^{2}
$$

and the free boundary is a $C^{1}(n-1)$-dimensional manifold in a neighbourhood of $X_{0}$.

The regularity $C^{1}$ can then be improved by Kinderlehrer, Nirenberg results [16], and gives $C^{\infty}$ regularity for an obstacle problem where the elliptic operator has $C^{\infty}$ coefficients. It is also possible to get similar results with analyticity of the solutions when the coefficients are analytic.

\subsection{More general blow-up limits}

We now recall a result which characterizes the limits of some more general blow-up sequences where the origin moves with the scaling.

Lemma 2.2 (General Blow-up Limits, [8]). - Let

$$
u^{\varepsilon}(X)=\frac{u_{\varepsilon}\left(X_{\varepsilon}+\varepsilon X\right)}{\varepsilon^{2}}
$$

where $u_{\varepsilon}$ is a sequence of solutions to

$$
\left\{\begin{array}{l}
\Delta u_{\varepsilon}=f_{\varepsilon} \geqslant 1 \quad \text { on } \quad\left\{u_{\varepsilon}>0\right\} \cap \Omega_{\varepsilon} \\
u_{\varepsilon} \geqslant 0 \text { on } \Omega_{\varepsilon} \quad \text { and }\left|D^{2} u_{\varepsilon}\right|_{L^{\infty}\left(\Omega_{\varepsilon}\right)} \leqslant M
\end{array}\right.
$$

with $\left|f_{\varepsilon}\right|_{C^{0, \alpha}\left(\Omega_{\varepsilon}\right)} \leqslant M$. We assume that $u_{\varepsilon}\left(X_{\varepsilon}\right)=0$ and that $\frac{1}{\varepsilon} d\left(X_{\varepsilon}, \partial \Omega_{\varepsilon}\right) \geqslant$ $r>0$ as $\varepsilon \rightarrow 0$. Then up to extraction of a convergent subsequence $\left(\varepsilon^{\prime}\right)$, we get $u^{\varepsilon^{\prime}} \longrightarrow u^{0} \quad$ uniformly on compact sets of $\Omega^{0}$ 
On the regularity of a free boundary for a nonlinear obstacle problem

for some open set $\Omega^{0}$ and where $u^{0}$ is convex and satisfies

$$
\left\{\begin{array}{lll}
\Delta u^{0}=f_{0}(0) \geqslant 1 \text { on } & \left\{u^{0}>0\right\} \cap \Omega^{0} \\
u^{0} \geqslant 0 \text { on } \Omega^{0} & \text { and } & \left|D^{2} u^{0}\right|_{L^{\infty}\left(\Omega^{0}\right)} \leqslant M
\end{array}\right.
$$

Moreover either

i) the interior of the coincidence set of the blow-up limit is empty:

$$
\left\{u^{0}=0\right\}^{0}=\emptyset
$$

Or

ii) the interior of the coincidence set of the blow-up limit satisfies

$$
\left\{u^{0}=0\right\}^{0} \neq \emptyset
$$

and 0 is a regular point for $u^{0}$ and also for all $u^{\varepsilon^{\prime}}$ with $\varepsilon^{\prime}$ small enough.

Another useful result is the following nondegeneracy property of the solution:

LEMMA 2.3 (Nondegeneracy, [8]). - Let u be a solution to problem (2.1) and $0 \in \overline{\{u>0\}}$. If $B_{r}(0) \subset \Omega$, then

$$
\sup _{B_{r}(0)}(u(X)-u(0)) \geqslant \frac{r^{2}}{2 n}
$$

Proof of lemma 2.3. - Apply the maximum principle to $w(X)=u(X)-$ $u(0)-\frac{1}{2 n}|X|^{2}$ in $B_{r}(0) \cap\{u>0\}$.

\section{A bound on the second derivatives}

In this section we will prove the following result

Proposition 3.1 (Control near the fixed boundary $\partial \Omega$ ). - Under the assumptions of theorem 1.2, let us define $\varepsilon=\sqrt{2\left(\frac{\lambda_{0}-\lambda}{\lambda}\right)}$. Then there exist constants $C, c>0$ such that for all $\lambda \in\left[0, \lambda_{0}\right]$ we have

$$
\begin{gathered}
u_{\lambda}(X)-\lambda \geqslant c \varepsilon^{2} \text { on }\{X \in \Omega, \quad \operatorname{dist}(X, \partial \Omega) \leqslant c \varepsilon\} \\
\left|\nabla u_{\lambda}(X)\right| \leqslant C \varepsilon \text { on } \Omega \\
-295-
\end{gathered}
$$


and for all $\delta \in(0,1]$

$$
\left|D^{2} u_{\lambda}(X)\right| \leqslant C / \delta^{2} \quad \text { on } \quad\{X \in \Omega, \quad \operatorname{dist}(X, \partial \Omega) \geqslant c \varepsilon \delta\}
$$

Moreover we have

$$
\operatorname{div}\left(F^{\prime}\left(\left|\nabla u_{\lambda}\right|^{2}\right) \nabla u_{\lambda}\right)=u_{\lambda} 1_{\left\{u_{\lambda}>\lambda\right\}} \quad \text { on } \quad \Omega
$$

where for the function $u_{\lambda} \geqslant \lambda$ we define

$$
1_{\left\{u_{\lambda}>\lambda\right\}}(X)=\left\{\begin{array}{lll}
1 & \text { if } & u_{\lambda}(X)>\lambda \\
0 & \text { if } & u_{\lambda}(X)=\lambda
\end{array}\right.
$$

Remark 3.2. - For a smooth $\Omega$, some $L^{\infty}$ bounds on the second derivatives are given in [6] for fixed $\lambda$. Here we need to precise the dependence of the constants as $\lambda$ goes to $\lambda_{0}$. The exterior sphere condition gives a control (3.1) from below on $u_{\lambda}$, and with the help of Harnack inequality we get the $L^{\infty}$ bounds (3.3) on the second derivatives up to the case $\lambda=\lambda_{0}$. Because the fixed boundary $\partial \Omega$ is not smooth here, the bound (3.3) on the second derivatives goes to infinity when the point reaches the fixed boundary $\partial \Omega$ $($ case $\delta=0)$.

We consider the minimizer $u_{\lambda}$ of the convex energy

$$
E(u)=\int_{\Omega} F\left(|\nabla u|^{2}\right)+u^{2}
$$

on the convex set

$$
K_{\lambda}=\left\{u \in H^{1}(\Omega), \quad u \geqslant \lambda \quad \text { on } \Omega, \quad u=\lambda_{0} \quad \text { on } \partial \Omega\right\}
$$

We first prove that the minimizer $u_{\lambda}$ satisfies the following Euler-Lagrange equation

LEMMA 3.3 (Euler-Lagrange equation). -

$$
\operatorname{div}\left(F^{\prime}\left(\left|\nabla u_{\lambda}\right|^{2}\right) \nabla u_{\lambda}\right)=u_{\lambda} 1_{\left\{u_{\lambda}>\lambda\right\}} \quad \text { on } \quad \Omega
$$

Although this result seems natural, we do not know any references where it is proved (except in the linear case). We give a complete proof below.

Proof of lemma 3.3. - Let

$$
\begin{aligned}
(s)^{+}= & \left\{\begin{array}{ccc}
s & \text { if } & s>0 \\
0 & \text { if } & s \leqslant 0
\end{array}\right. \\
& -296-
\end{aligned}
$$


Then the minimization of $E$ on $K_{\lambda}$ is equivalent to the minimization of the convex energy

$$
E_{\lambda}(u)=\int_{\Omega} F\left(|\nabla u|^{2}\right)+\left((u-\lambda)^{+}+\lambda\right)^{2}
$$

on the convex set

$$
K=\left\{u \in H^{1}(\Omega), \quad u=\lambda_{0} \quad \text { on } \quad \partial \Omega\right\}
$$

Because $u_{\lambda}$ is the minimizer of $E_{\lambda}$ on $K$, we have for every $\varphi \in C_{0}^{\infty}(\Omega)$ and $t \in[0,1]:$

$$
E_{\lambda}\left(u_{\lambda}+t \varphi\right) \geqslant E_{\lambda}\left(u_{\lambda}\right)
$$

Then Lebesgue's dominated convergence theorem gives

$$
\begin{aligned}
0 \leqslant & \lim _{t \rightarrow 0}\left(\frac{E_{\lambda}\left(u_{\lambda}+t \varphi\right)-E_{\lambda}\left(u_{\lambda}\right)}{t}\right) \\
= & \int_{\Omega} 2 F^{\prime}\left(\left|\nabla u_{\lambda}\right|^{2}\right) \nabla u_{\lambda} \nabla \varphi \\
& \quad+2 u_{\lambda}\left(\varphi \operatorname{sgn}^{+}\left(u_{\lambda}-\lambda\right)+\varphi^{+}\left(1-\operatorname{sgn}^{+}\left(u_{\lambda}-\lambda\right)\right)\right)
\end{aligned}
$$

where

$$
\operatorname{sgn}^{+}(s)=\left\{\begin{array}{lll}
1 & \text { if } & s>0 \\
0 & \text { if } & s \leqslant 0
\end{array}\right.
$$

Considering $\varphi$ and $-\varphi$ we get that $\operatorname{div}\left(F^{\prime}\left(\left|\nabla u_{\lambda}\right|^{2}\right) \nabla u_{\lambda}\right) \in L^{\infty}(\Omega)$. Using the regularity theory for elliptic equations (see [21]) we deduce that $u \in C_{\text {loc }}^{1, \alpha}(\Omega)$. Consequently $\left\{u_{\lambda}>\lambda\right\}$ is an open set and the Euler-Lagrange equation is satisfied on this open set. Furthermore a classical argument using the nondegeneracy lemma 2.3 proves that the Lebesgue measure of the free boundary $\partial\left\{u_{\lambda}=\lambda\right\}$ is zero. This implies the full Euler-Lagrange equation. This ends the proof of lemma 3.3.

Let us recall that when $\Omega$ is smooth, there exists a constant $C_{0}>0$ such that for each $\lambda \in\left[0, \lambda_{0}\right]$ we have the following properties (see Brézis, Kinderlehrer [6]):

$$
\left|\nabla u_{\lambda}(X)\right| \leqslant C_{0} \quad \text { on } \quad \Omega
$$

$$
\begin{gathered}
u \in C_{l o c}^{1,1}(\Omega) \\
-297-
\end{gathered}
$$


In a first case we will prove proposition 3.1 assuming $(\mathrm{H} 1)-(\mathrm{H} 2)$, and in a second case we will justify these assumptions.

Case A: we assume (H1)-(H2) and that $\partial \Omega$ is smooth

\section{Step 1: proof of (3.1)}

We will build a subsolution $u_{0}$ such that (for some point $X_{\varepsilon}$ which will be made precise below)

$$
\frac{u_{\lambda}(X)-\lambda}{\lambda} \geqslant \varepsilon^{2} u_{0}\left(\frac{\left|X-X_{\varepsilon}\right|}{\varepsilon}\right) \text { for } \frac{\left|X-X_{\varepsilon}\right|}{\varepsilon} \in\left[r_{0}, r_{0}+\tau_{0}\right]
$$

with $\varepsilon=\sqrt{2\left(\frac{\lambda_{0}-\lambda}{\lambda}\right)}$.

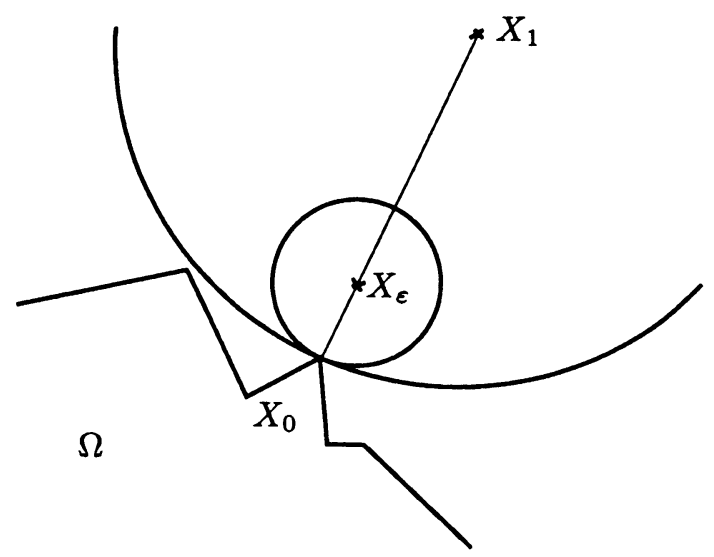

Figure 1. - Construction of a subsolution outside the ball $B_{\left|X_{0}-X_{\varepsilon}\right|}\left(X_{\varepsilon}\right)$

For some $\tau_{0}>0$, we consider a solution $u_{0}$ of

$$
\left\{\begin{array}{l}
\Delta u_{0}=\mu>1 \text { on } B_{r_{0}+\tau_{0}}(0) \backslash B_{r_{0}}(0) \\
u_{0}=\frac{1}{2} \text { on } \partial B_{r_{0}}(0) \\
u_{0}=0 \text { on } \partial B_{r_{0}+\tau_{0}}(0)
\end{array}\right.
$$

By symmetry we have $u_{0}(X)=u_{0}(|X|)$. Let us recall that for each point $X_{0} \in \partial \Omega$, there exists $X_{1} \in \mathbf{R}^{n}$, such that $B_{r_{0}}\left(X_{1}\right)$ is included in $\mathbf{R}^{n} \backslash \Omega$ and 
is tangent to $\partial \Omega$ at $X_{0}$. Now considering the function $u_{\lambda}$ at a scale close to the fixed boundary $\partial \Omega$ we introduce the point $X_{\varepsilon}=X_{0}+\varepsilon\left(X_{1}-X_{0}\right)$ and the following function (see figure 1)

$$
w^{\varepsilon}(X)=\frac{u_{\lambda}\left(X_{\varepsilon}+\varepsilon X\right)-\lambda}{\lambda \varepsilon^{2}}
$$

which satisfies on $\frac{\Omega-X_{\varepsilon}}{\varepsilon}$ :

$$
\left\{\begin{array}{l}
A_{\varepsilon}\left(w^{\varepsilon}\right) \leqslant 1 \\
0 \leqslant w^{\varepsilon} \leqslant \frac{1}{2}
\end{array}\right.
$$

where the quasilinear elliptic partial differential operator $A_{\varepsilon}$ is defined in (4.1).

Moreover for a good choice of $\mu>1, \tau_{0}>0$, we have on $B_{r_{0}+\tau_{0}}(0) \backslash B_{r_{0}}(0)$ :

$$
\left\{\begin{array}{l}
A_{\varepsilon}\left(u_{0}\right) \geqslant 1 \\
0 \leqslant u_{0} \leqslant \frac{1}{2}
\end{array}\right.
$$

Then by the Maximum Principle (see Berestycki, Nirenberg [3]), we can slide $u_{0}$ below $w^{\varepsilon}$ and we get

$$
w^{\varepsilon} \geqslant u_{0} \quad \text { on } \quad B_{r_{0}+\tau_{0}}(0) \backslash B_{r_{0}}(0)
$$

This is equivalent to (3.4) whose we deduce (3.1). This ends the proof of step 1.

Step 2: proof of (3.2): estimate on the gradient : $\left|\nabla u_{\lambda}\right| \leqslant \lambda \varepsilon\left|u_{0}^{\prime}\left(r_{0}\right)\right|$

We first remark that a straightforward consequence of step 1 is that

$$
\limsup _{X \rightarrow \partial \Omega}\left(\frac{\lambda_{0}-u_{\lambda}}{\operatorname{dist}(X, \partial \Omega)}\right) \leqslant \lambda \varepsilon\left|u_{0}^{\prime}\left(r_{0}\right)\right|
$$

From the fact that $u=$ constant $=\lambda_{0}$ on $\partial \Omega$, we deduce that $|\nabla u| \leqslant$ $\lambda \varepsilon\left|u_{0}^{\prime}\left(r_{0}\right)\right|$ on $\partial \Omega$. Now the estimate on the gradient comes from the fact that the gradient is maximal on the boundary $\partial \Omega$. For the convenience of the reader we recall this classical argument.

For $u=u_{\lambda}$, we have

$$
\begin{aligned}
a_{i j}(\nabla u) u_{i j} & =u \text { on } \Omega \backslash\{u=\lambda\} \\
& -299-
\end{aligned}
$$


where $a_{i j}(p)=F^{\prime}\left(|p|^{2}\right) \delta_{i j}+2 F^{\prime \prime}\left(|p|^{2}\right) p_{i} p_{j}$. Let us take $v=\partial_{\xi} u$ where $\xi \in \mathbf{S}^{n-1}$. Then

$$
a_{i j} v_{i j}+b_{k} v_{k}=v \quad \text { on } \quad \Omega \backslash\{u=\lambda\}
$$

where $b_{k}=\left(a_{i j}\right)_{p_{k}}^{\prime} \cdot u_{i j}$. The Maximum Principle implies that $v=\partial_{\xi} u$ is maximal on $\partial \Omega \cup \partial\{u=\lambda\}$. Taking all directions $\xi \in \mathbf{S}^{n-1}$ we deduce that $|\nabla u|$ is maximal on $\partial \Omega$, because $\nabla u=0$ on $\partial\{u=\lambda\}$.

This ends the proof of step 2.

\section{Step 3: proof of (3.3)}

Let

$$
w(X)=\frac{u_{\lambda}(\varepsilon X)-\lambda}{\lambda \varepsilon^{2}}
$$

Then

$$
\left\{\begin{array}{l}
A_{\varepsilon}(w)=1 \text { on }\{w>0\} \\
0 \leqslant w \leqslant \frac{1}{2}
\end{array}\right.
$$

where the operator $A_{\varepsilon}$ is defined in (4.1). Let $Y_{0} \in \frac{\Omega}{\varepsilon}$ such that $\operatorname{dist}\left(Y_{0}, \frac{\partial \Omega}{\varepsilon}\right) \geqslant c$. We will prove a bound on $\left|D^{2} w\left(Y_{0}\right)\right|$. To this end we will apply the method of Alt and Phillips [1], using the following Harnack inequality of Krylov, Safonov for non-divergence operator (a similar Harnack inequality for divergence operator is also applicable, see Gilbarg, Trudinger [15]):

THEOREM 3.4 (Harnack inequality for non-divergence operators; [7]). - If

$$
\left\{\begin{array}{l}
a_{i j} v_{i j}=f \text { on } B_{1} \subset \mathbf{R}^{n} \\
v \geqslant 0 \text { on } B_{1}
\end{array}\right.
$$

and for the matrix $a=\left(a_{i j}\right)$

$$
0<c_{0} \leqslant a \leqslant C_{0}
$$

then there exists a constant $C=C\left(n, C_{0}, c_{0}\right)>0$ such that

$$
\sup _{B_{\frac{1}{2}}} v \leqslant C\left(\inf _{B_{\frac{1}{2}}} v+|f|_{L^{n}\left(B_{1}\right)}\right)
$$

We will also use the following interior estimate: 
On the regularity of a free boundary for a nonlinear obstacle problem

Theorem 3.5 (Interior estimate,[15]). - Let us assume that

$$
a_{i j} v_{i j}+c v=f \quad \text { on } \quad B_{r} \subset \mathbf{R}^{n}
$$

and for the matrix $a=\left(a_{i j}\right)$

$$
0<c_{0} \leqslant a
$$

If for some $\alpha \in(0,1)$ there exists a constant $C_{0}>0$ such that

$$
\left|a_{i j}\right|_{L^{\infty}\left(B_{r}\right)}+r^{\alpha}\left[a_{i j}\right]_{\alpha ; B_{r}}+r^{2}|c|_{L^{\infty}\left(B_{r}\right)}+r^{2+\alpha}[c]_{\alpha ; B_{r}} \leqslant C_{0}
$$

where $[\cdot]_{\alpha ; B_{r}}$ is defined by

$$
[g]_{\alpha ; B_{r}}=\sup _{x, y \in B_{r}, x \neq y}\left(\frac{|g(x)-g(y)|}{|x-y|^{\alpha}}\right)
$$

Then

$$
r^{2}\left|D^{2} v\right|_{L^{\infty}\left(B_{\frac{r}{2}}\right)} \leqslant C\left(|v|_{L^{\infty}\left(B_{r}\right)}+r^{2}|f|_{L^{\infty}\left(B_{r}\right)}+r^{2+\alpha}[f]_{\alpha ; B_{r}}\right)
$$

for some constant $C=C\left(n, \alpha, C_{0}, c_{0}\right)>0$.

Let $w_{r}(X)=w\left(Y_{0}+r X\right)$. Applying Harnack inequality theorem 3.4 to $w_{r}$ we get

$$
\sup _{B_{\frac{r}{2}}\left(Y_{0}\right)} w \leqslant C\left(\inf _{B_{\frac{r}{2}}\left(Y_{0}\right)} w+r^{2}\right)
$$

Let

$$
\rho=\sqrt{\frac{w\left(Y_{0}\right)}{2 C}}
$$

i) Case $\rho<c \delta$.

Then $Y_{0}$ is close to $\{w=0\}$ and $\rho$ can be arbitrarily small. We apply Harnack inequality (3.5) with $r=\rho$ and we get

$$
0<w\left(Y_{0}\right) \leqslant \sup _{B_{\frac{\rho}{2}}\left(Y_{0}\right)} w \leqslant 2 C \inf _{B_{\frac{\rho}{2}}\left(Y_{0}\right)} w
$$

Let us remark that we have (see theorem 6.1, p. 281 of Ladyshenskaya, Ural'tseva [18])

$$
[w]_{\alpha ; B_{1}} \leqslant C
$$

where the constant $C$ has the following dependence

$$
C=C\left(n, \alpha,|w|_{L^{\infty}\left(B_{2}\right)}, F, \lambda_{0}, r_{0}\right)>0 .
$$


Then applying theorem 3.5, we deduce that

$$
r^{2}\left|D^{2} w\right|_{L^{\infty}\left(B_{\frac{r}{2}\left(Y_{0}\right)}\right)} \leqslant C\left(|w|_{L^{\infty}\left(B_{r}\left(Y_{0}\right)\right)}+r^{2}\right)
$$

With the choice $r=\rho$, this implies

$$
\left|D^{2} w\left(Y_{0}\right)\right| \leqslant C
$$

ii) case $\rho \geqslant c \delta$.

We apply the previous interior estimate with $r=c \delta$. Using the fact that $|w| \leqslant \frac{1}{2}$, we find

$$
\left|D^{2} w\left(Y_{0}\right)\right| \leqslant C / \delta^{2}
$$

iii) Conclusion :

$$
\left.\left|D^{2} u_{\lambda}\right| \leqslant C / \delta^{2} \quad \text { on } \quad\{X \in \Omega, \quad \operatorname{dist}(X, \partial \Omega) \geqslant c \varepsilon \delta)\right\}
$$

i.e. (3.3) is proved.

\section{Case B: justification of (H1)-(H2)}

Here we consider a general Lipschitz bounded open set $\Omega$ satisfying assumptions (A1), (A2) of theorem 1.2. We can mollify this open set $\Omega$ such that it gives a bigger and smooth open set $\Omega^{\eta}$ where $\eta$ is the mollification parameter such that $\Omega^{\eta}=\Omega$ for $\eta=0$. This smooth open set $\Omega^{\eta}$ still satisfies assumptions (A1), (A2) uniformly in $\eta$ small enough. We can in particular consider the minimizer $u_{\lambda}^{\eta}$ of the energy

$$
\left.E^{\eta}(u)=\int_{\Omega^{\eta}} F\left(|\nabla u|^{2}\right)+u^{2}\right)
$$

on the convex set

$$
K_{\lambda}^{\eta}=\left\{u \in H^{1}\left(\Omega^{\eta}\right), \quad u \geqslant \lambda \quad \text { on } \Omega^{\eta}, \quad u=\lambda_{0} \quad \text { on } \partial \Omega^{\eta}\right\}
$$

This minimizer $u_{\lambda}^{\eta}$ satisfies (H1)-(H2), and then (3.1),(3.2),(3.3).

Taking the limit $\eta \rightarrow 0$, we can extract (by Ascoli-Arzela theorem) a convergent subsequence $u_{\lambda}^{\eta} \rightarrow u$ such that $u$ still satisfies (3.1),(3.2),(3.3). We have the

Lemma 3.6. - The limit $u$ is the minimizer $u_{\lambda}$ of the energy $E$ on $K_{\lambda}$.

This ends the proof of proposition 3.1. 
Proof of lemma 3.6. - Let us recall that by (3.2), $u_{\lambda}^{\eta}$ is bounded in $W^{1, \infty}$ uniformly in $\eta$ small enough. Let

$$
\tilde{u}_{\lambda}=\left\{\begin{array}{lll}
\lambda_{0} & \text { on } & \Omega^{\eta} \backslash \Omega \\
u_{\lambda} & \text { on } & \Omega
\end{array}\right.
$$

By construction, we have

$$
E^{\eta}\left(\tilde{u}_{\lambda}\right) \geqslant E^{\eta}\left(u_{\lambda}^{\eta}\right)
$$

At the limit $\eta=0$, we get

$$
E\left(u_{\lambda}\right) \geqslant E(u)
$$

The uniqueness of the minimizer $u_{\lambda}$ proves that $u=u_{\lambda}$. This ends the proof of the lemma 3.6.

\section{Regularity of the free boundary near $\partial \Omega$ : proof of theorem 1.2}

We will prove theorem 1.2, thanks to Caffarelli result (lemma 2.2) applied to a particular blow-up sequence.

Case $F(q)=q$

If theorem 1.2 is false, then there exist a sequence of reals $\varepsilon_{n}=\sqrt{2\left(\frac{\lambda_{0}-\lambda^{n}}{\lambda^{n}}\right)}$ $\rightarrow 0$ and a sequence of singular points $X_{\lambda^{n}} \in \partial\left\{u_{\lambda^{n}}=\lambda^{n}\right\}$. Because of proposition 3.1, we have $\operatorname{dist}\left(X_{\lambda^{n}}, \partial \Omega\right)>c \varepsilon_{n}$. Then we define

$$
w^{\varepsilon_{n}}(X)=\frac{u_{\lambda^{n}}\left(X_{\lambda^{n}}+\varepsilon_{n} X\right)-\lambda^{n}}{\lambda^{n} \varepsilon_{n}^{2}}
$$

We have

$$
\left\{\begin{array}{l}
\Delta w^{\varepsilon_{n}}=1+\varepsilon_{n}^{2} w^{\varepsilon_{n}} \quad \text { on } \quad\left\{w^{\varepsilon_{n}}>0\right\} \\
0 \leqslant w^{\varepsilon_{n}} \leqslant \frac{1}{2}
\end{array}\right.
$$

Now from proposition 3.1 we have the following $L^{\infty}$ bound on the second derivatives:

$$
\left|D^{2} w^{\varepsilon_{n}}(X)\right| \leqslant C \text { for } \operatorname{dist}\left(X_{\lambda^{n}}+\varepsilon_{n} X, \partial \Omega\right) \geqslant c \varepsilon_{n}
$$

Consequently from lemma 2.2 , there exists a subsequence which converges to a convex function $w^{0}$ defined on $\Omega_{0}$, where $\Omega_{0}$ is the limit of the sets 
$\frac{1}{\varepsilon_{n}}\left(\Omega-X_{\lambda^{n}}\right)$ (for an extracted subsequence). Moreover $w^{0}$ satisfies

$$
\left\{\begin{array}{l}
\Delta w^{0}=1 \quad \text { on } \quad\left\{w^{0}>0\right\} \\
0 \leqslant w^{0} \leqslant \frac{1}{2} \text { and }\left|D^{2} w^{0}(X)\right| \leqslant C \text { for } \operatorname{dist}\left(X, \partial \Omega_{0}\right) \geqslant c
\end{array}\right.
$$

Because $\Omega$ satisfies an interior cone condition (A2), $\Omega_{0}$ inherits the same property. Moreover because we have made a blow-up close to the fixed boundary $\partial \Omega$, we deduce that $\Omega_{0}$ contains an infinite cone $\mathcal{C}_{0}$ with a nonempty interior. Now we have two cases (see lemma 2.2):

i) the interior of the coincidence set of the blow-up limit is empty, and then the closure $\overline{\left\{w^{0}>0\right\}}$ contains the cone $\mathcal{C}_{0}$. It is then sufficient to take a ball $B_{r} \subset \mathcal{C}_{0}$ with $r$ large enough such that (by the nondegeneracy lemma 2.3)

$$
\sup _{B_{r}} w^{0} \geqslant \frac{r^{2}}{2 n}
$$

which is in contradiction with $0 \leqslant w^{0} \leqslant \frac{1}{2}$.

ii) the interior of the coincidence set of the blow-up limit is not empty, and then 0 is a regular point for $w^{0}$, and also a regular point for $w^{\varepsilon_{n}^{\prime}}$ for $\varepsilon_{n}^{\prime}$ small enough. This means that $X_{\lambda^{n}}$ are regular points for $u_{\lambda^{n}}$. Contradiction.

\section{Case $F$ general}

In this case we introduce the operator (for $\varepsilon=\sqrt{2\left(\frac{\lambda_{0}-\lambda}{\lambda}\right)}$ )

$$
A_{\varepsilon}(w)=a\left(\left(\frac{\lambda_{0}}{1+\frac{\varepsilon^{2}}{2}}\right) \varepsilon \nabla w\right) D^{2} w-\varepsilon^{2} w
$$

where $a(p)=F^{\prime}\left(p^{2}\right) I d+2 F^{\prime \prime}\left(p^{2}\right) p \otimes p$. Then we have

$$
\left\{\begin{array}{l}
A_{\varepsilon_{n}}\left(w^{\varepsilon_{n}}\right)=1 \quad \text { on } \quad\left\{w^{\varepsilon_{n}}>0\right\} \\
0 \leqslant w^{\varepsilon_{n}} \leqslant \frac{1}{2}
\end{array}\right.
$$

A generalization of previous Caffarelli results to more general linear elliptic operators

$$
\begin{aligned}
L= & \alpha_{i j} \partial_{i j}+\beta_{i} \partial_{i}+\gamma \\
& -304-
\end{aligned}
$$


is available in [8]. This allows us to get similar results in the same way for our general case.

This ends the proof of theorem 1.2.

\section{Stability: proof of theorem 1.4}

In this section we will prove theorem 1.4 on stability. A similar result is already known in the linear case (see for instance the book of Rodrigues [22] for general results of stability). In our case we use the approach of Caffarelli [8].

Proof of theorem 1.4. - Let us assume that the theorem is false. Then for a compact set $\mathcal{K} \subset \subset \mathcal{K}^{*}$ we can find a sequence $\left(\lambda^{n}\right)_{n}$ such that $\lambda^{n} \rightarrow \lambda^{*}$ and a sequence of singular points $\left(X_{\lambda^{n}}\right)_{n}$ of the free boundaries $\partial\left\{u_{\lambda^{n}}=\lambda^{n}\right\} \cap \mathcal{K}$. Up to extract a subsequence we can assume

$$
X_{\lambda^{n^{\prime}}} \longrightarrow X_{\lambda^{*}} \in\left\{u_{\lambda^{*}}=\lambda^{*}\right\} \cap \mathcal{K}
$$

where we have used the continuity of the map

$$
\lambda \longmapsto u_{\lambda}
$$

The continuity of this map is a consequence of the $L^{\infty}$ bound on the gradient of $u_{\lambda}$ uniformly in $\lambda$ (see (3.2)). This continuity easily follows by a classical argument from Ascoli-Arzela theorem, and the uniqueness of the solutions $u_{\lambda}$ for each $\lambda$.

Let us recall that for $\varepsilon=\sqrt{2\left(\frac{\lambda_{0}-\lambda}{\lambda}\right)}$ we have (the operator $A_{\varepsilon}$ is defined in $(4.1))$

$$
A_{\varepsilon}\left(w_{\lambda}\right)=1 \quad \text { on } \quad\left\{w_{\lambda}>0\right\}
$$

where for some point $X_{\lambda} \in \Omega$ :

$$
w_{\lambda}(X)=\frac{u_{\lambda}\left(X_{\lambda}+\varepsilon X\right)-\lambda}{\lambda \varepsilon^{2}}
$$

Using the adaptation of the nondegeneracy lemma 2.3 (see Caffarelli [8]) for general linear elliptic operators, we get the existence of a constant $c_{0}>0$ such that

$$
\sup _{B_{r}\left(X_{\lambda^{n}}\right)}\left(u_{\lambda^{n}}(X)-\lambda^{n}\right) \geqslant c_{0} r^{2}
$$

Then at the limit we get

$$
\sup _{B_{r}\left(X_{\lambda^{*}}\right)}\left(u_{\lambda^{*}}(X)-\lambda^{*}\right) \geqslant c_{0} r^{2}
$$


which proves that $X_{\lambda^{*}} \in \partial\left\{u_{\lambda^{*}}=\lambda^{*}\right\}$. In particular because $X_{\lambda^{*}}$ is a regular point for $u_{\lambda^{*}}$, i.e. 0 is a regular point for $w_{\lambda^{*}}$, we get that the blow-up sequence

$$
w_{\lambda^{*}}^{\delta}(X)=\frac{w_{\lambda^{*}}(\delta X)}{\delta^{2}}
$$

converges (up to extraction of a subsequence) to a blow-up limit of regular type (see theorem 2.1; for an extension to general linear elliptic operators, see Caffarelli $[8])$ :

$$
w_{\lambda^{*}}^{0}(X)=\frac{1}{2}\left(\max \left(<X, \nu_{X_{\lambda^{*}}}>, 0\right)\right)^{2}
$$

We realize that the origin 0 is obviously a regular point of $w_{\lambda^{*}}^{0}$. Finally we can consider the other blow-up sequence:

$$
w_{\lambda^{n}}^{\delta^{n}}(X)=\frac{w_{\lambda^{n}}\left(\delta^{n} X\right)}{\left(\delta^{n}\right)^{2}}
$$

Because for $\delta^{n}=\delta$ fixed and $\lambda^{n} \rightarrow \lambda^{*}$, this sequence of functions converges to $w_{\lambda^{*}}^{\delta}$, we see that we can choose a sequence $\left(\delta^{n}\right)_{n}$ slowly decreasing to zero such that

$$
w_{\lambda^{n}}^{\delta^{n}} \longrightarrow w_{\lambda^{*}}^{0}
$$

Then applying an adaptation of lemma 2.2 (see Caffarelli [8]) still true for general linear elliptic operators, we deduce from the fact that 0 is a regular point for the blow-up limit of $w_{\lambda^{n}}^{\delta^{n}}$, that 0 is also a regular point for $w_{\lambda^{n}}^{\delta^{n}}$ for $n$ large enough. This means that $X_{\lambda^{n}}$ is a regular point for $u_{\lambda^{n}}$. Contradiction. This ends the proof of theorem 1.4.

\section{Hausdorff measure of the free boundary: proof of theorem 1.5}

In this section we give the proof of theorem 1.5, which is an adaptation of a method of Caffarelli presented in the linear case in $[9,19]$. We perform the proof in two steps.

\section{Step 1}

For the function $u=u_{\lambda}$, let

$$
O^{\eta}=\{X \in \Omega, \quad|\nabla u(X)|<\eta \quad \text { and } \quad u(X)>\lambda\}
$$

For a function $u \geqslant \lambda$, we note

$$
\begin{aligned}
1_{\{u>\lambda\}}(X)= & \left\{\begin{array}{lll}
1 & \text { if } & u(X)>\lambda \\
0 & \text { if } & u(X)=\lambda
\end{array}\right. \\
& -306-
\end{aligned}
$$


LEMma 6.1 (Estimate in a neighbourhood of the free boundary). - If

$$
\begin{cases}\nabla \cdot\left(F^{\prime}\left(|\nabla u|^{2}\right) \nabla u\right)=u 1_{\{u>\lambda\}} & \text { on } \quad \Omega \\ u \geqslant \lambda>0 & \text { on } \quad \partial \Omega \\ \left|D^{2} u(X)\right| \leqslant M & \text { on } \quad\{X \in \Omega, \quad \operatorname{dist}(X, \partial \Omega) \geqslant c \varepsilon\}\end{cases}
$$

then for all compact $\mathcal{K} \subset\{X \in \Omega, \quad \operatorname{dist}(X, \partial \Omega) \geqslant c \varepsilon\}$ such that $\partial \mathcal{K}$ is $C^{1}$, there is a constant $C=C(M)$, such that

$$
\left|O^{\eta} \cap \mathcal{K}\right| \leqslant \eta C \lambda^{-2}\left(|\mathcal{K}|+\mathcal{H}^{n-1}(\partial \mathcal{K})\right)
$$

where $|\mathcal{K}|$ is the volume of $\mathcal{K}$ and $\mathcal{H}^{n-1}(\partial \mathcal{K})$ is the $(n-1)$ dimensional Hausdorff measure of its perimeter.

Remark 6.2 (The Hausdorff measure). - Let us recall the definition of the Hausdorff measure. If $U$ is a set, let

$$
\operatorname{diam}(U)=\sup _{X, X^{\prime} \in U}\left|X^{\prime}-X\right|
$$

Then for $s \geqslant 0$ and a set $A$ let

$$
\mathcal{H}_{\delta}^{s}(A)=c_{s} \inf _{\left\{\left\{U^{i}\right\}_{i}, A \subset \cup_{i} U^{i}, \operatorname{diam} U^{i} \leqslant \delta\right\}} \sum_{i}\left(\operatorname{diam} U^{i}\right)^{s}
$$

which is a nondecreasing function of $\delta$. Then the $s$-dimensional Hausdorff measure is

$$
\mathcal{H}^{s}(A)=\lim _{\delta \rightarrow 0} \mathcal{H}_{\delta}^{s}(A)
$$

The constant $c_{s}$ must be chosen such that the Hausdorff measure coincides with the Lebesgue measure of $\mathbf{R}^{s}$ if $s \in \mathbf{N}$.

Proof of lemma 6.1. - Because $F^{\prime} \in C^{1,1}$, we have $h_{i} \in C^{0,1}$ where

$$
h_{i}= \begin{cases}-\eta & \text { if } F^{\prime} \nabla_{i} u \leqslant-\eta \\ F^{\prime} \cdot \nabla_{i} u & \text { if }\left|F^{\prime} \nabla_{i} u\right| \leqslant \eta \\ \eta & \text { if } F^{\prime} \nabla_{i} u \geqslant \eta\end{cases}
$$

We note $X_{i}$ the vector field defined by $X_{i}=\nabla_{i}\left(F^{\prime} \nabla u\right) \in L^{\infty}$. Then the Stokes formula gives :

$$
\begin{gathered}
\int_{\mathcal{K}} \nabla h_{i} \cdot X_{i}=\int_{\partial \mathcal{K}} h_{i}\left(X_{i} \cdot n\right)-\int_{\mathcal{K}} h_{i}\left(\nabla \cdot X_{i}\right) \\
-307-
\end{gathered}
$$


But $\nabla \cdot X_{i}=\nabla_{i}\left(\nabla \cdot\left(F^{\prime} \nabla u\right)\right)=\nabla_{i} u$ on $\{u>\lambda\}$, and $h_{i}=0$ on $\{u=\lambda\}$. Then

$$
\int_{O^{n} \cap \mathcal{K}} \nabla\left(F^{\prime} \nabla_{i} u\right) \cdot \nabla_{i}\left(F^{\prime} \nabla u\right) \leqslant \eta C(M)\left(|\mathcal{K}|+\mathcal{H}^{n-1}(\partial \mathcal{K})\right)
$$

But

$$
\nabla\left(F^{\prime} \nabla_{i} u\right) \cdot \nabla_{i}\left(F^{\prime} \nabla u\right)=\left[\nabla_{i}\left(F^{\prime} \nabla_{i} u\right)\right]^{2}+\sum_{k \neq i}\left[F^{\prime} D_{i k}^{2} u\right]^{2}+O\left(|\nabla u|^{2}\right)
$$

and

$$
\left|\int_{O^{\eta} \cap \mathcal{K}} O\left(|\nabla u|^{2}\right)\right| \leqslant \eta C(M)|\mathcal{K}|
$$

Making the sum $\sum_{i}$, we get

$$
\left.\int_{O^{\eta} \cap \mathcal{K}} \sum_{i}\left(\nabla_{i}\left(F^{\prime} \nabla_{i} u\right)\right)\right)^{2} \leqslant \eta C(M)\left(|\mathcal{K}|+\mathcal{H}^{n-1}(\partial \mathcal{K})\right)
$$

But

$$
\left.\sum_{i}\left(\nabla_{i}\left(F^{\prime} \nabla_{i} u\right)\right)\right)^{2} \geqslant\left(\frac{\nabla \cdot\left(F^{\prime} \nabla u\right)}{2}\right)^{2} \geqslant \frac{u^{2}}{4} \geqslant \frac{\lambda^{2}}{4}
$$

and then we get the expected result.

\section{Step 2}

The Hausdorff measure is bounded from above by:

$$
\mathcal{H}^{n-1}(\Gamma) \leqslant \lim _{\eta \rightarrow 0} \inf _{\left\{B_{\eta}\left(Y_{i}\right)\right\}} \frac{1}{\eta} \sum_{i}\left|B_{\eta}\left(Y_{i}\right)\right|
$$

where $\Gamma=\partial\{u=\lambda\}$ is the free boundary, and where $\left\{B_{\eta}\left(Y_{i}\right)\right\}_{i}$ is a recovering of $\Gamma$ by balls of center $Y_{i}$ on $\Gamma$ and of radius $\eta$.

From proposition 3.1, we know that

$$
u(X)-\lambda \geqslant c \varepsilon^{2} \quad \text { while } \quad \operatorname{dist}(X, \partial \Omega)<c \varepsilon \quad \text { where } \quad \varepsilon=\sqrt{2\left(\frac{\lambda_{0}-\lambda}{\lambda}\right)}
$$

which in particular implies

$$
\begin{aligned}
\operatorname{dist}(\{u= & \lambda\}, \partial \Omega) \geqslant c \varepsilon \\
& -308-
\end{aligned}
$$


Now starting from a point $Y_{i}$ on $\partial\{u=\lambda\}$ we have from (3.3)

$$
u(X)-\lambda \leqslant \frac{1}{2} C\left|X-Y_{i}\right|^{2} \quad \text { while } \operatorname{dist}(X, \partial \Omega) \geqslant c \varepsilon
$$

Therefore we get

$$
\operatorname{dist}\left(B_{\eta}\left(Y_{i}\right), \partial \Omega\right) \geqslant c \varepsilon \quad \text { while } \quad \frac{1}{2} C \eta^{2}<c \varepsilon^{2}
$$

i.e. for $\eta$ small enough. Then for such $\eta$ we have

$B_{\eta}\left(Y_{i}\right) \cap\{u>\lambda\} \quad \subset \quad B_{\eta}\left(Y_{i}\right) \cap\{u>\lambda, \quad|\nabla u| \leqslant C \eta\} \quad \subset \quad B_{\eta}\left(Y_{i}\right) \cap O^{C \eta}$

From the nondegeneracy lemma 2.3, we deduce the existence of a real $\gamma \in$ $(0,1)$ such that

$$
\left|B_{\eta}\left(Y_{i}\right) \cap\{u>\lambda\}\right| \geqslant \gamma\left|B_{\eta}\left(Y_{i}\right)\right|
$$

As a consequence we get

$$
\left|B_{\eta}\left(Y_{i}\right)\right| \leqslant \gamma^{-1}\left|B_{\eta}\left(Y_{i}\right) \cap O^{C \eta}\right|
$$

Thus

$$
\begin{aligned}
\eta^{-1} \sum_{i}\left|B_{\eta}\left(Y_{i}\right)\right| & \leqslant \eta^{-1} \gamma^{-1} \sum_{i}\left|B_{\eta}\left(Y_{i}\right) \cap O^{C \eta}\right| \\
& \leqslant \eta^{-1} \gamma^{-1} \int_{\Omega} \sum_{i} 1_{B_{\eta}\left(Y_{i}\right)} 1_{O^{C} \eta} \\
& \leqslant \eta^{-1} \gamma^{-1} \sup \left(\sum_{i} 1_{B_{\eta}\left(Y_{i}\right)}\right) \int_{\Omega} 1_{O^{C \eta}} \\
& \leqslant \eta^{-1} \gamma^{-1} \sup \left(\sum_{i} 1_{B_{\eta}\left(Y_{i}\right)}\right)\left|O^{C \eta}\right| \\
& \leqslant \gamma^{-1} C_{n} C^{\prime} \lambda^{-2}\left(\left|\mathcal{K}_{\varepsilon}\right|+\mathcal{H}^{n-1}\left(\partial \mathcal{K}_{\varepsilon}\right)\right)
\end{aligned}
$$

where we have used the fact that we can always use locally finite recovering $\left\{B_{\eta}\left(Y_{i}\right)\right\}_{i}$ such that $\sum_{i} 1_{B_{\eta}\left(Y_{i}\right)} \leqslant C_{n}$ where the constant only depends on the dimension $n$. On the other hand we have applied lemma 6.1 introducing a smooth compact set $\mathcal{K}_{\varepsilon}$ such that

$$
\mathcal{K}_{\varepsilon} \quad \subset \quad\{X \in \Omega, \quad 2 c \varepsilon \geqslant \operatorname{dist}(X, \partial \Omega) \geqslant c \varepsilon\}
$$

In fact $\mathcal{K}_{\varepsilon}$ can be seen as a smooth approximation of $\partial \Omega$. Consequently we get

$$
\mathcal{H}^{n-1}(\Gamma) \leqslant C
$$

where the constant $C$ only depends on $\Omega, \lambda_{0}$ and $F$, and is uniform with respect to $\lambda \in\left[0, \lambda_{0}\right]$. This proves theorem 1.5. 
Régis Monneau

\section{Acknowledgements}

The author would like to thank A. Bonnet, S.J. Chapman and J.F. Rodrigues for stimulating discussions and helpful comments.

\section{Bibliography}

[1] Alt (H.W.), Phillips (D.). - A free boundary problem for semilinear elliptic equations, J. Reine Angew. Math. 368, p. 63-107 (1986).

[2] Berestycki (H.), Bonnet (A.), Chapman (S.J.). - A Semi-elliptic System Arising in the Theory of type-II Superconductivity, Comm. Appl. Nonlinear Anal. 1, p. 1-21 (1994).

[3] Berestycki (H.), Nirenberg (L.). - On the method of moving planes and the sliding method, Bol. Soc. Braseleira Mat. (N.S.) 22, 1-37 (1991).

[4] Bonnet (A.), Chapman (S.J.), Monneau (R.). - Convergence of Meissner minimisers of the Ginzburg-Landau energy of superconductivity as kappa tends to infinity, SIAM J. Math. Anal. 31 (6), p. 1374-1395 (2000).

[5] Bonnet (A.), Monneau (R.). - Distribution of vortices in a type II superconductor as a free boundary problem: Existence and regularity via Nash-Moser theory, Interfaces and Free Boundaries 2, p. 181-200 (2000).

[6] BRÉzis (H.), Kinderlehrer (D.). - The Smoothness of Solutions to Nonlinear Variational Inequalities, Indiana Univ. Math. J. 23 (9), p. 831-844 (1974).

[7] Cabré (X.), Caffarelli (L.A.). - Fully Nonlinear Elliptic Equations, Colloquium Publications. Amer. Math. Soc. 43 (1995).

[8] Caffarelli (L.A.). - Compactness Methods in Free Boundary Problems, Comm. Partial Differential Equations 5 (4), p. 427-448 (1980).

[9] Caffarelli (L.A.). - A remark on the Hausdorff measure of a free boundary, and the convergence of coincidence sets, Boll. Un. Mat. Ital. A 18 (5), p. 109-113 (1981).

[10] Caffarelli (L.A.). - Free boundary problem in higher dimensions, Acta Math. 139, p. 155-184 (1977).

[11] Caffarelli (L.A.). - The Obstacle Problem revisited, J. Fourier Anal. Appl. 4, p. 383-402 (1998).

[12] Caffarelli (L.A.), Salazar (J.), Shahgholian (H.). - Free Boundary Regularity for a Problem Arising in Superconductivity, Arch. Ration. Mech. Anal. 171 (1), p. 115-128 (2004).

[13] Chapman (S.J.), Rubinstein (J.), Schatzman (M.). - A Mean-field Model of Superconducting vortices, European J. Appl. Math. 7, p. 97-111 (1996).

[14] Friedman (A.). - Variational Principles and Free Boundary Problems, Pure and applied mathematics, ISSN 0079-8185, a Wiley-Interscience publication, (1982).

[15] Gilbarg (D.), Trudinger (N.S.). - Elliptic Partial Differential Equations of Second Order, Springer-Verlag (1997).

[16] Kinderlehrer (D.), Nirenberg (L.). - Regularity in free boundary problems, Ann. Scuola Norm. Sup. Pisa Cl. Sci 4, p. 373-391 (1977).

[17] D. Kinderlehrer, G. Stampacchia. - An Introduction to Variational Inequalities and Their Applications, Academic Press, New York, (1980). 
On the regularity of a free boundary for a nonlinear obstacle problem

[18] Ladyshenskaya (O.A.), URAL'tseva (N.N.). - Linear and Quasilinear Elliptic Equations, New York: Academic Press, (1968).

[19] Lin (F.H.). - An unpublished course at Courant Institute of Mathematical Sciences, (1990).

[20] Monneau (R.). - On the Number of Singularities for the Obstacle Problem in Two Dimensions, J. of Geometric Analysis 13 (2), p. 359-389 (2003).

[21] Morrey (C.B.). - Multiple Integrals in the Caculus of Variations, SpringerVerlag, Berlin-Heidelberg-New York, (1966).

[22] J.F. Rodrigues. - Obstacle Problems in Mathematical Physics, North-Holland, (1987).

[23] Sandier (E.), Serfaty (S.). - A Rigorous Derivation of a Free-Boundary Problem Arising in Superconductivity, Annales Scientifiques de l'ENS 33, p. 561-592, $(2000)$.

[24] Sandier (E.), Serfaty (S.). - On the Energy of Type-II Superconductors in the Mixed Phase, Reviews in Mathematical Physics 12, No 9, p. 1219-1257, (2000).

[25] Serfaty (S.). - Stable Configurations in Superconductivity: Uniqueness, Multiplicity and Vortex-Nucleation, Archive for Rational Mechanics and Analysis 149, p. 329-365 (1999).

[26] WeISS (G.S.). - A homogeneity improvement approach to the obstacle problem, Invent. math. 138, p. 23-50 (1999). 